\title{
Insecticidal activity of different fractions of petroleum ether extract of Zingiber cassumunar rhizome against Tribolium castaneum
}

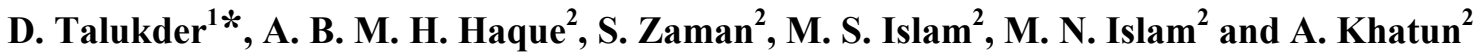 \\ ${ }^{1}$ BCSIR Laboratories, Rajshahi, Rajshahi-6206, Banglasesh. \\ ${ }^{2}$ Department of Chemistry, University of Rajshahi, Rajshahi-6205, Banglasesh.
}

\begin{abstract}
An experiment was carried out to investigate the efficacy of contact toxicity of different fractions of petroleum ether extract of Zinziber cassumunar Roxb. rhizome against Tribolium castaneum. Seventy-eight different fractions of petroleum ether extract were obtained from column chromatography. Elutes having the similar TLC behavior were combined in eight fractions and were named as: $\mathrm{MN}-1, \mathrm{MN}-2, \mathrm{MN}-3$, $\mathrm{MN}-4, \mathrm{MN}-5, \mathrm{MN}-6, \mathrm{MN}-7$ and $\mathrm{MN}-8$. Four separate fractions were collected from the MN-1 fraction by preparative thin layer chromatography. These four parts were washed with petroleum ether, chloroform, ethyl acetate and methanol. Thereafter, these were separated by small column and designated as: $\mathrm{M}_{1} \mathrm{~N}_{1}, \mathrm{M}_{1} \mathrm{~N}_{2}, \mathrm{M}_{1} \mathrm{~N}_{3}, \mathrm{M}_{1} \mathrm{~N}_{4}, \mathrm{M}_{2} \mathrm{~N}_{1}, \mathrm{M}_{2} \mathrm{~N}_{2}, \mathrm{M}_{2} \mathrm{~N}_{3}, \mathrm{M}_{2} \mathrm{~N}_{4}, \mathrm{M}_{3} \mathrm{~N}_{1}, \mathrm{M}_{3} \mathrm{~N}_{2}, \mathrm{M}_{3} \mathrm{~N}_{3}, \mathrm{M}_{3} \mathrm{~N}_{4}, \mathrm{M}_{4} \mathrm{~N}_{1}$ and $\mathrm{M}_{4} \mathrm{~N}_{2}$. Fractions of M1N2, $\mathrm{M}_{2} \mathrm{~N}_{2}, \mathrm{M}_{3} \mathrm{~N}_{2}$ and $\mathrm{M}_{4} \mathrm{~N}_{2}$ were found to be the most effective against the beetle T. castaneum after 24, 48 and 72 hours. However, some fractions exhibited the moderate effect and other fractions did not work against the beetle.
\end{abstract}

Keywords: Petroleum ether extracts; Z. cassumunar rhizome; Chromatography; Insecticidal activity; Tribolium castaneum adults

\section{Introduction:}

The presence of insect infestation in stored products always posed unique problems. There are more or less 200 stored grain and stored products attacking insects and mites species are found (Khanam et al., 2005). Among these, the red flour beetle, Tribolium castaneum (Coleoptera: Tenebrionidae) and the confused flour beetle, Tribolium confusum Coleoptera: Tenebrionidae) are serious pest of a great variety of stored products, which are cosmopolitan in distribution. Both the adults and larvae cause serious damage to stored wheat, maize and wheat flour. Several insecticides are used indiscriminately to control this pest. But indiscriminate use of chemical pesticides produced many serious problems, viz. genetic resistance of pest species, toxic residues, threat to wild life, etc. (Talukder et al., 2011). In fact this led a worldwide interest in the development of botanical pest control agent. The main advantages of botanicals are that, these can be produced easily by farmers and are potentially less expensive.

Zingiber cassumunar Roxb. commonly known as Bonada (Family: Zingiberaceae) is used in folklore remedies as a single plant or as component of herbal recipes for the treatment of inflammation, sprains, rheumatism, muscular pain wounds and also as mosquito repellent, a carminative, a mild laxative and an anti dysenteric agent in Bangladesh and many Asian countries (Bhuiyan et al., (2008). Z. cassumunar grows abundantly in Bangladesh. It is a herb with elongated leafy stem. Stem is 1.2 to $1.8 \mathrm{~cm}$ high. Leaves are sub sessile 23 to $35 \mathrm{~cm}$ oblong. Bhuiyan and Co-workers (Bhuiyan et al., 2008) identified 32 volatile constituents in the rhizome oil of $Z$. cassumunar. The main components in rhizome oil were triquinance-1,4-bis (methoxy) (26.47\%), Z-ocimene (21.97\% and terpinen-4-ol (18.45\%). Wanauppathamkul (2003) also reported the presence of Sabinene (25-45\%), $\gamma$-tepinene (5-10\%), $\alpha$-Tepinene (2-5\%), Terpinen-4-ol (25-45\%) and (E)-1-(3,4-dimethoxyphenyl) butadiene(DMPBD) (1-10\%) as active chemicals in the $Z$. cassumunar essential oil.

Several workers reported the chemical composition, anti-inflammatory, antimicrobial and insecticidal activity of Z. cassumunar (Sukatta et al., 2009; Giwanon et al., 2000; Pithayanukul et al., 2008; Thripathi 2008; Kamazeri et al., 2012; Yanbin Lu et al., 2005; Iswantini et al.,2011; Chairul, 2009 Khanam et al., 2008; Nugroho et al.,1996; Bandara et al., 2005; Talukder and Khanam, 2009; Somboom and Pimsamarn, 2011; Suthisut et al., 2011). These encouraged the authors to find out the effective fraction of this plant having insecticidal activities against stored product pests. Therefore, the following investigation was undertaken to evaluate the insecticidal activity of different fraction of petroleum ether extract of $Z$. cassumunar rhizome against $T$. castaneum.

\footnotetext{
*Corresponding author: E-mail : dtbcsir12@gmail.com
} 


\section{Materials and methods}

Stock culture of $T$. castaneum was maintained in plastic containers $(1200 \mathrm{~mL})$ and sub-cultures in beakers $(1000 \mathrm{~mL})$ with the food medium at $30^{\circ} \mathrm{C} \pm 0.5^{\circ} \mathrm{C}$ in an incubator. $\mathrm{A}$ standard mixture of whole-wheat flour with powdered dry yeast in a ratio of 19: 1 (Park, 1961) was used as food medium.

The rhizomes of Bonada, Z. cassumunar were procured from different areas of Rajshahi, Bangladesh. The rhizomes were chopped off into small pieces and dried in a shade. Finally, it was dried in an oven at $40^{\circ} \mathrm{C}$. After drying these parts were crushed (200mesh) by using a cyclotech grinding machine. After crushing, the plant materials were extracted in a soxhlet apparatus separately with petroleum ether, acetone and methanol. The extraction process was carried out by refluxing the solvent for twenty hours $\left(A_{1}\right)$. The solvents were evaporated in rotary vacuum evaporator at $40^{\circ} \mathrm{C}$ under reduced pressure and the petroleum ether, acetone and methanol extracts were collected in small reagent bottle and preserved at $4^{\circ} \mathrm{C}$ in a refrigerator. The concentrated peroleum ether extract was mixed with a small amount of column grade Silica gel (70-230 mesh, E-MERCK) maintaing the ratio as: concentrated mass : Silica gel $=2: 1$ and dried in air. After drying, the mixture was powdered in a mortar. This powder was then ready for fractionation by column.

The petroleum ether extract was prepared for column chromatography using mobile phase toluene, chloroform, ethyle acetate and methanol. The column was elute first with $100 \%$ toluene and increasing amount of chloroform and then increasing amount of ethyl acetate, finally methanol (Table I). Elute were collected in an amount of about $50 \mathrm{~mL}$ in a series of conical flask. Elute of similar behaviour were

\section{Table I. Solvent used for eluting the column chromatography}

\begin{tabular}{cccccc}
\hline Toluene $(\mathrm{mL})$ & $\begin{array}{c}\text { Chloroform } \\
(\mathrm{mL})\end{array}$ & $\begin{array}{c}\text { Ethyl acetate } \\
(\mathrm{mL})\end{array}$ & Methanol $(\mathrm{mL})$ & Total $(\mathrm{mL})$ & Fraction \\
\hline $600(100 \%)$ & $0(0 \%)$ & $0(0 \%)$ & $0(0 \%)$ & 600 & $1-9$ \\
$190(95 \%)$ & $10(5 \%)$ & $0(0 \%)$ & $0(0 \%)$ & 200 & $10-14$ \\
$270(90 \%)$ & $30(10 \%)$ & $0(0 \%)$ & $0(0 \%)$ & 300 & $15-19$ \\
$240(80 \%)$ & $60(20 \%)$ & $0(0 \%)$ & $0(0 \%)$ & 300 & $20-25$ \\
$280(70 \%)$ & $120(30 \%)$ & $0(0 \%)$ & $0(0 \%)$ & 400 & $26-32$ \\
$180(60 \%)$ & $120(40 \%)$ & $0(0 \%)$ & $0(0 \%)$ & 300 & $33-37$ \\
$50(50 \%)$ & $50(50 \%)$ & $0(0 \%)$ & $0(0 \%)$ & 100 & $38-40$ \\
$0(0 \%)$ & $100(100 \%)$ & $0(0 \%)$ & $0(0 \%)$ & 100 & $41-42$ \\
$0(0 \%)$ & $196(98 \%)$ & $4(2 \%)$ & $0(0 \%)$ & 200 & $43-45$ \\
$0(0 \%)$ & $475(95 \%)$ & $25(5 \%)$ & $0(0 \%)$ & 500 & $46-54$ \\
$0(0 \%)$ & $90(90 \%)$ & $10(10 \%)$ & $0(0 \%)$ & 100 & $55-57$ \\
$0(0 \%)$ & $160(80 \%)$ & $40(20 \%)$ & $0(0 \%)$ & 200 & $58-60$ \\
$0(0 \%)$ & $140(70 \%)$ & $60(30 \%)$ & $0(0 \%)$ & 200 & $61-66$ \\
$0(0 \%)$ & $50(50 \%)$ & $50(50 \%)$ & $0(0 \%)$ & 100 & $67-69$ \\
$0(0 \%)$ & $392(98 \%)$ & $0(0 \%)$ & $8(2 \%)$ & 400 & $70-76$ \\
$0(0 \%)$ & $190(95 \%)$ & $0(0 \%)$ & $10(5 \%)$ & 200 & $78-80$ \\
$0(0 \%)$ & $180(90 \%)$ & $0(0 \%)$ & $20(10 \%)$ & 200 & $81-84$ \\
\hline
\end{tabular}


combined together based on Thin layer chromatography (TLC) analysis. There were seventy-eight serially fraction obtained from column chromatography (CC) which were combined in eight fraction were designated as: $\mathrm{MN}-1$, MN-2, MN-3, MN-4, MN-5, MN-6, MN-7 and MN-8 (Table II.). Thin layer chromatography of the above eight fractions were observed (Table III.). Then fraction, MN-1 was subjected to preparative thin layer chromatography (PTLC) using toluene: Chloroform (7:1) solvent system. The separated bands were visualized by the use of UV light $(350 \mathrm{~nm})$. Four sharp bands were marked with a pin and were collected in different $100 \mathrm{~mL}$ beakers, which were numbered

Table II. Designation of fractions of petroleum ether extracts having similar TLC behaviour, obtained after column

\begin{tabular}{|c|c|c|}
\hline Fraction & Fraction No. & Designation \\
\hline 1 & $4-12$ & $\mathrm{MN}-1$ \\
\hline 2 & $19-24$ & $\mathrm{MN}-2$ \\
\hline 3 & $28-34$ & $\mathrm{MN}-3$ \\
\hline 4 & $37-40$ & $\mathrm{MN}-4$ \\
\hline 5 & $44-48$ & MN-5 \\
\hline 6 & $51-55$ & MN-6 \\
\hline 7 & $59-62$ & MN-7 \\
\hline 8 & $72-78$ & MN-8 \\
\hline
\end{tabular}

Table III. TLC behavior of the fractions obtained from column chromatography of petroleum ether extract

\begin{tabular}{|c|c|c|}
\hline Fraction & Solvent system & Observation \\
\hline $\mathrm{MN}-1$ & Toluene : chloroform & $\begin{array}{l}\text { Four spots }\left(\mathrm{R}_{\mathrm{f}} 0.30,0.56,0.69 \text {, }\right. \\
0.9) \text {. }\end{array}$ \\
\hline $\mathrm{MN}-2$ & Toluene : chloroform & $\begin{array}{l}\text { Four spots }\left(\mathrm{R}_{\mathrm{f}} 0.28,0.47,0.59 \text {, }\right. \\
0.80) \text {. }\end{array}$ \\
\hline $\mathrm{MN}-3$ & Toluene : ethyl acetate $(8: 2)$ & $\begin{array}{l}\text { One spot }\left(\mathrm{R}_{\mathrm{f}} 0.48\right) \text { with tailing } \\
\text { from the baseline. }\end{array}$ \\
\hline $\mathrm{MN}-4$ & Toluene : ethyl acetate $(8: 2)$ & Two spots with tailing. \\
\hline $\mathrm{MN}-5$ & Toluene : ethyl acetate $(5: 2)$ & One spot $\left(\mathrm{R}_{\mathrm{f}} 0.58\right)$. \\
\hline MN-6 & Chloroform : Ethyl acetate $(5: 2)$ & $\begin{array}{l}\text { Three different spots with } \\
\text { long tailing. }\end{array}$ \\
\hline $\mathrm{MN}-7$ & Chloroform : Ethyl acetate $(5: 2)$ & Tailing present, no clear spot. \\
\hline MN-8 & Ethyl acetate : Methanol $(5: 2)$ & Long tailing present. \\
\hline
\end{tabular}


Table IV. $\chi^{2}$ value regression equation, $\mathrm{LD}_{50}$ and $95 \%$ confident limits of rhizome extract of Zingiber cassumunar against Tribolium castaneum adult after 24, 48 and 72 hours of treatment

\begin{tabular}{ccccccc}
\hline & $\begin{array}{c}\text { Duration } \\
\text { after } \\
\text { treatment }\end{array}$ & $\begin{array}{c}\text { Solvent } \\
\text { used }\end{array}$ & $\begin{array}{c}\chi^{2} \text { for } \\
\text { heterogeneity }\end{array}$ & $\begin{array}{c}\text { Regression } \\
\text { equation }\end{array}$ & $\begin{array}{c}\text { LD }_{50} \\
\mu \mathrm{cm}^{-2}\end{array}$ & $\begin{array}{c}\text { confinement limit } \\
\text { Lower }\end{array}$ \\
upper
\end{tabular}

Table V. TLC behaviour of different fraction of petroleum ether extracts of Zingiber cassumunar rhizome

\begin{tabular}{|c|c|c|c|c|c|c|c|c|c|c|c|c|c|c|}
\hline \multirow{2}{*}{ Solvent System } & \multicolumn{14}{|c|}{$R_{f}$ vallues of different fraction } \\
\hline & $\mathrm{M}_{1} \mathrm{~N}_{1}$ & $\mathrm{M}_{1} \mathrm{~N}_{2}$ & $\mathrm{M}_{1} \mathrm{~N}_{3}$ & $\mathrm{M}_{1} \mathrm{~N}_{4}$ & $\mathrm{M}_{2} \mathrm{~N}_{1}$ & $\mathrm{M}_{2} \mathrm{~N}_{2}$ & $\mathrm{M}_{2} \mathrm{~N}_{3}$ & $\mathrm{M}_{2} \mathrm{~N}_{4}$ & $\mathrm{M}_{3} \mathrm{~N}_{1}$ & $\mathrm{M}_{3} \mathrm{~N}_{2}$ & $\mathrm{M}_{3} \mathrm{~N}_{3}$ & $\mathrm{M}_{3} \mathrm{~N}_{4}$ & $\mathrm{M}_{4} \mathrm{~N}_{1}$ & $\mathrm{M}_{4} \mathrm{~N}_{2}$ \\
\hline Toluene:Chloroform (7:1) & 0.42 & & & & & & & & $\begin{array}{l}0.30 \\
0.33 \\
\text { and } \\
0.39\end{array}$ & & & & & \\
\hline Toluene: chloroform $(8: 1)$ & & 0.70 & & & & 0.67 & & & & & & & & \\
\hline n-hesane: Ethyleacetate(7:1) & & & 0.80 & & & & & & & & & & & $\begin{array}{l}0.73, \\
0.79 \\
\text { and } \\
0.83\end{array}$ \\
\hline Toluene: Methanol (8:1) & & & & 0.87 & $\begin{array}{l}0.67(a) \\
0.49(b)\end{array}$ & & & 0.54 & & & & & & \\
\hline $\begin{array}{l}\text { n-hexane : Ethyl acetate (8: } \\
\text { 1) }\end{array}$ & & & & & & & 0.75 & & & & & & & \\
\hline $\begin{array}{l}\text { Toluene : Chloroform (7: } \\
\text { 2). }\end{array}$ & & & & & & & & & & 0.67 & & & & \\
\hline $\begin{array}{l}\text { n-hexane : Ethyl acetate }(9 \text { : } \\
\text { 1) }\end{array}$ & & & & & & & & & & & 0.69 & & & \\
\hline Toluene : Methanol $(8: 2)$ & & & & & & & & & & & & 0.74 & & \\
\hline Toluene : Chloroform $(7: 3)$ & & & & & & & & & & & & & 0.68 & \\
\hline
\end{tabular}


as M-1, M-2, M-3 and M-4. These four part were washed with petroleum ether, chloroform, ethyl acetate and methanol respectively and separated by small colum and designated as $\mathrm{M}_{1} \mathrm{~N}_{1}, \mathrm{M}_{1} \mathrm{~N}_{2}, \mathrm{M}_{1} \mathrm{~N}_{3}, \mathrm{M}_{1} \mathrm{~N}_{4}, \mathrm{M}_{2} \mathrm{~N}_{1}, \mathrm{M}_{2} \mathrm{~N}_{2}, \mathrm{M}_{2} \mathrm{~N}_{3}$,
$\mathrm{M}_{2} \mathrm{~N}_{4}, \mathrm{M}_{3} \mathrm{~N}_{1}, \mathrm{M}_{3} \mathrm{~N}_{2}, \mathrm{M}_{3} \mathrm{~N}_{3}, \mathrm{M}_{3} \mathrm{~N}_{4}, \mathrm{M}_{4} \mathrm{~N}_{1}, \mathrm{M}_{4} \mathrm{~N}_{2}$ etc. and the content were dried.TLC behaviour of these fourteen fractions were observed (Table V). The schematic pathway of fraction described as follows.

\section{FLOW CHART OF PLANT EXTRACTION}

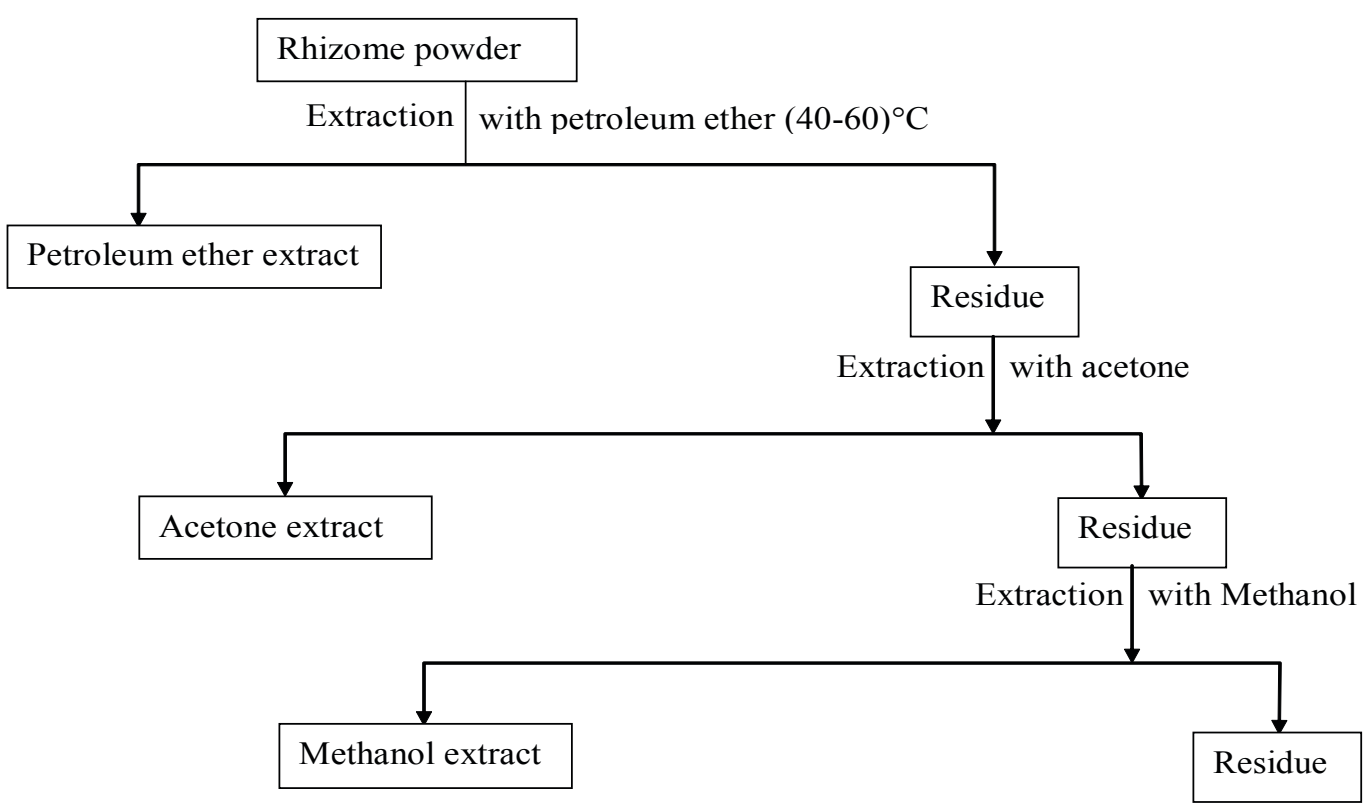

SCHEMATIC PATHWAY OF DIFFERENT FRACTION OF PRTROLEUM ETHER EXTRACT OF Z. cassumUnar

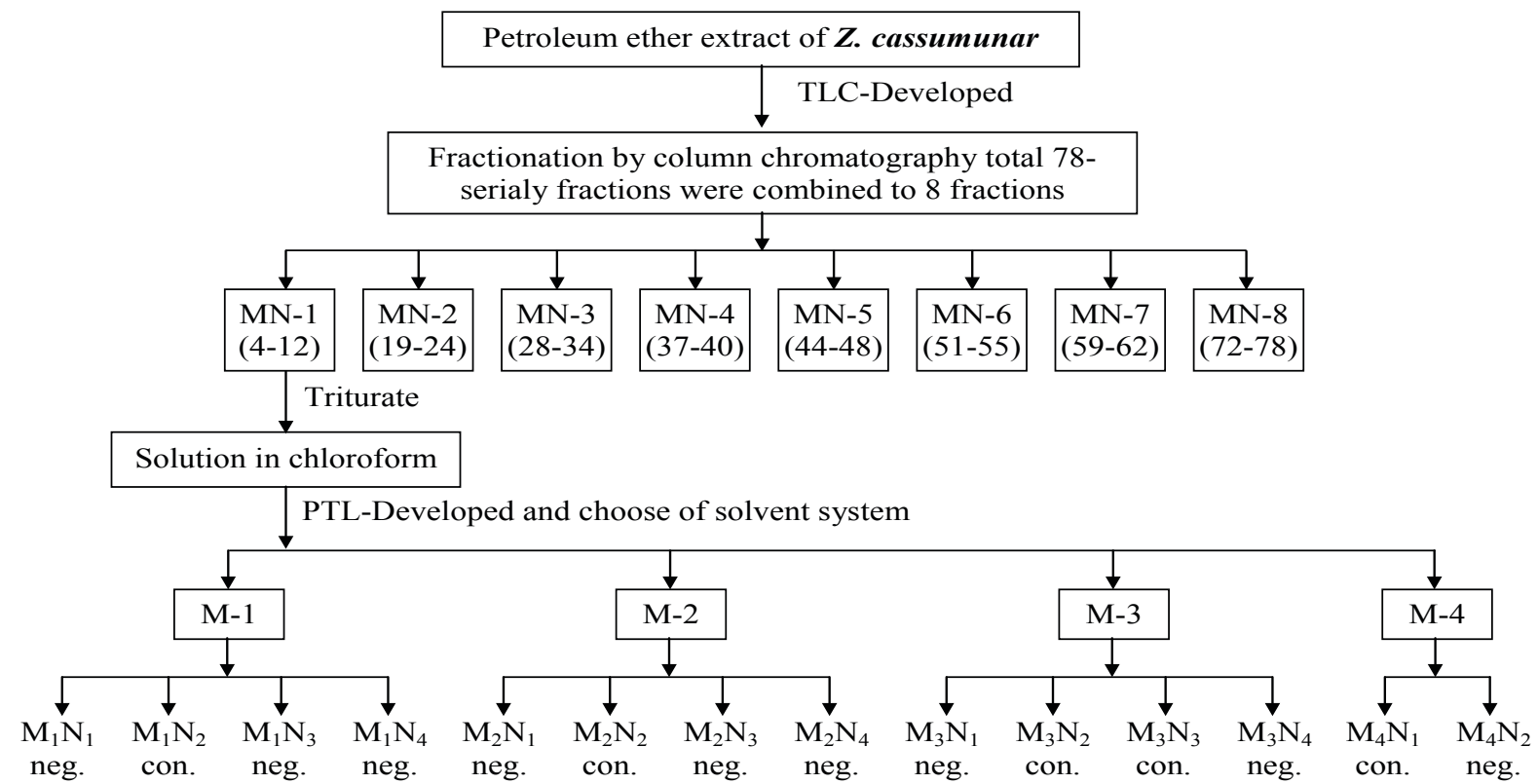

neg. $\rightarrow$ Negligible

con. $\rightarrow$ Considerable 


\section{Experiment setting}

Residual film technique (Busvine, 1971) was used to test the mortality rate of the larvae and adults of T. castaneum. The doses were prepared by the mixing the requisite quantities of different solvent extract and different fraction of petroleum ether extract of $Z$. cassumunar with $10 \mathrm{~mL}$ acetone or methanol. Methanol was used in the case of methanol extract because these extracts do not dissolve properly in acetone. The experimental doses were 78.60, 157.19, $314.38,471.57$ and $628.76 \mathrm{mg} / \mathrm{cm}^{2}$ for all the solvents. The doses were prepared by mixing the requisite quantities (5000, 10000, 20000, 30000 and 40000mg per petridish) of extracted materials with $1 \mathrm{~mL}$ acetone or methanol. For testing the mortality, each dose with $1 \mathrm{~mL}$ solvent was dropped on a petridish $(9.5 \mathrm{~cm}$ dia.). After drying, three petridishes were taken each with forty adult insects (one to two week old adult beetles of $T$. castaneum) considered as three replications. Other three petridishes contained only solvent and same number of insects considered as control. The experiment was performed at $30^{\circ} \mathrm{C} \pm 0.5^{\circ} \mathrm{C}$. The doses were calculated by measuring the weight of extracted materials $(\mathrm{mg})$ in $01 \mathrm{ml}$ of the solvent divided by the surface area of the petridish and it is converted in to $\mathrm{mg} / \mathrm{cm}^{2}$. Mortality was assessed after 24, 48 and 72 hours of the treatment applied. The percentage of mortality was corrected using Abbott's formula (Abbott, 1925) and $\mathrm{LD}_{50}$ values were determined by probit analysis (Busvine, 1971).

\section{Result and discussion}

The result of the contact toxicity, $\mathrm{LD}_{50}$, regression equation, and fiducial limits, due to the effect of different solvent extract of $Z$. cassumunar against $T$. castaneum are summarized in Table, 4, 6-9. From the result, it can be seen that all the solvent extracts were exhibited toxic effect to the beetle, T. castaneum, (Table IV). However, the highest mortality was observed in petroleum ether extract at all the intervals. The $\mathrm{LD}_{50}$ values in case of petroleum ether extract were $225.91,102.34$ and $78.80 \mu \mathrm{g} / \mathrm{cm}^{2}$. The $\mathrm{LD}_{50}$ values of acetone extract were $2945.20,700.81$ and $454.75 \mu \mathrm{g} / \mathrm{cm}^{2}$. In case of methanol extract, these were 889.47, 767.94 and $297.96 \mu \mathrm{g} / \mathrm{cm}^{2}$ Their efficiency followed the order Petroleum ether $>$ Methanol $>$ Acetone.

Toxicity data of the different fraction of the petroleum ether extract against the adult T. castaneum were shown in table, 6. Among the four separated bands visualized by UV light and washed with petroleum ether fractions $\left(M_{1} N_{1}, M_{1} N_{2}\right.$, $\mathrm{M}_{1} \mathrm{~N}_{3}, \mathrm{M}_{1} \mathrm{~N}_{4}$ ) only $\mathrm{M}_{1} \mathrm{~N}_{1}$ fraction were found to be effective against $T$. castaneum, whereas other fractions $\left(\mathrm{M}_{1} \mathrm{~N}_{2}, \mathrm{M}_{1} \mathrm{~N}_{3}\right.$, $\mathrm{M}_{1} \mathrm{~N}_{4}$ ) did not show any effect against T.castaneum adult. The calculated $\mathrm{LD}_{50}$ values of $\mathrm{M}_{1} \mathrm{~N}_{1}$ fraction were 1220.96 ,
436.55 and $334.57 \mu \mathrm{g} / \mathrm{cm}^{2}$ after 24,48 and 72 hours expousure time respectively against $T$. castaneum.

Toxic effects due to the appliction of four separated bands visualized by UV light and washed with chloromorm fraction $\left(\mathrm{M}_{2} \mathrm{~N}_{1}, \mathrm{M}_{2} \mathrm{~N}_{2}, \mathrm{M}_{2} \mathrm{~N}_{3}\right.$ and $\left.\mathrm{M}_{2} \mathrm{~N}_{4}\right)$ against the adults $T$. castaneum were shown in table-7. Results demonstrate that all these different fractions $\left(\mathrm{M}_{2} \mathrm{~N}_{1}, \mathrm{M}_{2} \mathrm{~N}_{2}, \mathrm{M}_{2} \mathrm{~N}_{3}\right.$ and $\left.\mathrm{M}_{2} \mathrm{~N}_{4}\right)$ were effective against the $T$. castaneum adults at all the duration. $\mathrm{M}_{3} \mathrm{~N}_{2}$ fraction exhibited lowest $\mathrm{LD}_{50}$ values at 24, 48 and 72 hours treatment. However, $\mathrm{M}_{2} \mathrm{~N}_{2}$ fraction did not show any effect at 24 hours treatment. The $\mathrm{LD}_{50}$ values of $\mathrm{M}_{2} \mathrm{~N}_{3}$ fraction were $222.73,117.18$ and $100.32 \mu \mathrm{g} / \mathrm{cm}^{2}$ and $\mathrm{M}_{2} \mathrm{~N}_{4}$ fractions were $317.20,292.40$ and $233.29 \mu \mathrm{g} / \mathrm{cm}^{2}, \mathrm{M}_{2} \mathrm{~N}_{1}$ fraction were $358.34,277.51$ and $168.30 \mu \mathrm{g} / \mathrm{cm}^{2}$ after 24,48 and 72 hours exposure time respectively. The order of efficacy at 24hours was $\mathrm{M}_{2} \mathrm{~N}_{3}>\mathrm{M}_{2} \mathrm{~N}_{4}>\mathrm{M}_{2} \mathrm{~N}_{1}>\mathrm{M}_{2} \mathrm{~N}_{2}$ fraction. At 48 hours their efficacy followed the order as $\mathrm{M}_{2} \mathrm{~N}_{3}>$ $\mathrm{M}_{2} \mathrm{~N}_{1}>\mathrm{M}_{2} \mathrm{~N}_{4}>\mathrm{M}_{2} \mathrm{~N}_{2}$ fraction and 72 hours the efficacy followed the order as $\mathrm{M}_{2} \mathrm{~N}_{3}>\mathrm{M}_{2} \mathrm{~N}_{1}>\mathrm{M}_{2} \mathrm{~N}_{4}>\mathrm{M}_{2} \mathrm{~N}_{2}$ fraction.

Toxicity data of the four separate bands visualized by UV light and washed with ethylacetate fraction $\left(\mathrm{M}_{3} \mathrm{~N}_{1}, \mathrm{M}_{3} \mathrm{~N}_{2}\right.$, $\mathrm{M}_{3} \mathrm{~N}_{3}$ and $\mathrm{M}_{3} \mathrm{~N}_{4}$ ) against adult $T$. castaneum were shown in table-8. The results indicated that highest mortality was observed with $\mathrm{M}_{3} \mathrm{~N}_{3}$ fraction at 24, 48 and 72 hours treatment. However, other fractions $\left(\mathrm{M}_{3} \mathrm{~N}_{1}, \mathrm{M}_{3} \mathrm{~N}_{2}\right.$ and $\left.\mathrm{M}_{3} \mathrm{~N}_{4}\right)$ did not show any effect against $T$. castaneum at 24 hours exposure time. The $\mathrm{M}_{3} \mathrm{~N}_{4}$ fraction also did not show any effect at all the duration. The $\mathrm{LD}_{50}$ values of $\mathrm{M}_{3} \mathrm{~N}_{3}$ fraction were $415.47,185.22$ and $154.54 \mu \mathrm{g} / \mathrm{cm}^{2}$ after 24,48 and 72hours exposure time respectively. The $\mathrm{LD}_{50}$ values of $\mathrm{M}_{3} \mathrm{~N}_{1}$ fraction were 277.51 and $168.30 \mu \mathrm{g} / \mathrm{cm}^{2}, \mathrm{M}_{3} \mathrm{~N}_{2}$ fraction were 4563.90 and $357.08 \mu \mathrm{g} / \mathrm{cm}^{2}$ after 48 and 72 hours treatment respectively. After 48 and 72 hours treatment their efficacy followed the order $\mathrm{M}_{3} \mathrm{~N}_{3}>\mathrm{M}_{3} \mathrm{~N}_{1}>$ $\mathrm{M}_{3} \mathrm{~N}_{2}$.

Toxicity data of the four separate bands visualized by UV light and washed with methanol fraction $\left(\mathrm{M}_{4} \mathrm{~N}_{1}, \mathrm{M}_{4} \mathrm{~N}_{2}, \mathrm{M}_{4} \mathrm{~N}_{3}\right.$ and $\mathrm{M}_{4} \mathrm{~N}_{4}$ ) against $T$. castaneum adults are shown Table-9. Our results showed that only fractions, $\mathrm{M}_{3} \mathrm{~N}_{4}$ were exhibited the effective toxicity against $T$. castaneum adults whereas other fractions $\left(\mathrm{M}_{4} \mathrm{~N}_{1}, \mathrm{M}_{4} \mathrm{~N}_{2}\right.$ and $\left.\mathrm{M}_{4} \mathrm{~N}_{4}\right)$ did not show any effect at all the duration. The $\mathrm{LD}_{50}$ values of the $\mathrm{M}_{3} \mathrm{~N}_{4}$ fraction were $326.13,223.25$ and $151.21 \mu \mathrm{g} / \mathrm{cm}^{2}$ at 24,48 and 72 hours exposures time respectively.

Our findings are in accordance with the findings of Khamam and Co-workers (Khamam et al., 2006), who reported that petroleum ether extract of $Z$. cassumunar rhizome, Thevetia neriifolia root caused highest mortality than those of other solvent extracts, methanol and acetone, against $S$. oryzae. Khanam et al., 
Table VI. Relative toxicity of different separated bands visualized by UV light and washed with petroleum ether fraction of Zingiber cassumunar rhizome against Tribolium castaneum adults

\begin{tabular}{|c|c|c|c|c|c|c|c|}
\hline \multirow{2}{*}{$\begin{array}{l}\text { Hours } \\
\text { after } \\
\text { treatment }\end{array}$} & \multirow{2}{*}{$\begin{array}{l}\text { Plant materials } \\
\text { Zingiber cassumunar }\end{array}$} & \multirow[b]{2}{*}{ Fractions } & \multirow{2}{*}{$\begin{array}{l}\chi^{2} \text { values for } \\
\text { heterogeneity }\end{array}$} & \multirow{2}{*}{ Regression equation } & \multirow{2}{*}{$\begin{array}{l}\mathrm{LD}_{50} \\
\left(\mu \mathrm{g} / \mathrm{cm}^{2}\right)\end{array}$} & \multicolumn{2}{|c|}{ Fiducial limits } \\
\hline & & & & & & Lower & Upper \\
\hline \multirow{4}{*}{ 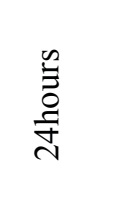 } & No. 1 Petroleum ether fraction & $\mathrm{M}_{1} \mathrm{~N}_{1}$ & 1.80 & $Y=-1.25+2.03 X$ & 1220.96 & 341.15 & 4369.75 \\
\hline & No. 2 Petroleum ether fraction & $\mathrm{M}_{1} \mathrm{~N}_{2}$ & - & - & - & - & - \\
\hline & No. 3 Petroleum ether fraction & $\mathrm{M}_{1} \mathrm{~N}_{3}$ & - & - & - & - & - \\
\hline & No. 4 Petroleum ether fraction & $\mathrm{M}_{1} \mathrm{~N}_{4}$ & - & - & - & - & - \\
\hline \multirow{4}{*}{$\begin{array}{l}\stackrel{\infty}{\Xi} \\
\stackrel{\Xi}{\Xi} \\
\stackrel{\infty}{+}\end{array}$} & No. 1 Petroleum ether fraction & $\mathrm{M}_{1} \mathrm{~N}_{1}$ & 2.15 & $Y=-2.04+2.67 X$ & 436.55 & 313.52 & 607.88 \\
\hline & No. 2 Petroleum ether fraction & $\mathrm{M}_{1} \mathrm{~N}_{2}$ & - & - & - & - & - \\
\hline & No. 3 Petroleum ether fraction & $\mathrm{M}_{1} \mathrm{~N}_{3}$ & - & - & - & - & - \\
\hline & No. 4 Petroleum ether fraction & $\mathrm{M}_{1} \mathrm{~N}_{4}$ & - & - & - & - & - \\
\hline \multirow{4}{*}{ 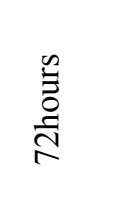 } & No. 1 Petroleum ether fraction & $\mathrm{M}_{1} \mathrm{~N}_{1}$ & 0.329 & $\mathrm{Y}=0.675+1.71 \mathrm{X}$ & 334.57 & 229.50 & 487.75 \\
\hline & No. 2 Petroleum ether fraction & $\mathrm{M}_{1} \mathrm{~N}_{2}$ & - & - & - & - & - \\
\hline & No. 3 Petroleum ether fraction & $\mathrm{M}_{1} \mathrm{~N}_{3}$ & - & - & - & - & - \\
\hline & No. 4 Petroleum ether fraction & $\mathrm{M}_{1} \mathrm{~N}_{4}$ & - & - & - & - & - \\
\hline
\end{tabular}

Table VII. Relative toxicity of different separated bands visualized by UV light and washed with chloroform fraction of Zingiber cassumunar rhizome against Tribolium castaneum adults

\begin{tabular}{|c|c|c|c|c|c|c|c|}
\hline \multirow{2}{*}{$\begin{array}{l}\text { Hours } \\
\text { after } \\
\text { treatment }\end{array}$} & \multirow{2}{*}{$\begin{array}{l}\text { Plant materials } \\
\text { cassumunar }\end{array}$} & \multirow{2}{*}{ Fractions } & \multirow{2}{*}{$\begin{array}{l}\chi^{2} \text { values for } \\
\text { heterogeneity }\end{array}$} & \multirow{2}{*}{ Regression equation } & \multirow{2}{*}{$\begin{array}{c}\mathrm{LD}_{50} \\
\left(\mu \mathrm{g} / \mathrm{cm}^{2}\right)\end{array}$} & \multicolumn{2}{|c|}{ Fiducial limits } \\
\hline & & & & & & Lower & Upper \\
\hline \multirow{5}{*}{$\begin{array}{l}\stackrel{\infty}{\Xi} \\
\stackrel{0}{7} \\
\stackrel{d}{d}\end{array}$} & No. 1 Chloroform fraction & $\mathrm{M}_{2} \mathrm{~N}_{1}$ & 0.175 & $\mathrm{Y}=-3.19+3.20 \mathrm{X}$ & 358.34 & 286.75 & 447.79 \\
\hline & No. 2 Chloroform fraction & $\mathrm{M}_{2} \mathrm{~N}_{2}$ & - & - & - & - & - \\
\hline & No. 3 Chloroform fraction & $\mathrm{M}_{2} \mathrm{~N}_{3}$ & 3.12 & $Y=-6.18+4.76 X$ & 222.73 & 198.96 & 249.33 \\
\hline & No. 4 Chloroform fraction & $\mathrm{M}_{2} \mathrm{~N}_{4}$ & 1.71 & $Y=-5.76+4.30 X$ & 317.20 & 273.69 & 367.63 \\
\hline & No. 1 Chloroform fraction & $\mathrm{M}_{2} \mathrm{~N}_{1}$ & 2.23 & $Y=-2.79+3.19 X$ & 277.51 & 233.19 & 330.25 \\
\hline \multirow{4}{*}{$\begin{array}{c}\stackrel{\infty}{\Xi} \\
\stackrel{0}{\Xi} \\
\stackrel{\infty}{+}\end{array}$} & No. 2 Chloroform fraction & $\mathrm{M}_{2} \mathrm{~N}_{2}$ & 0.57 & $Y=-2.64+2.89 X$ & 444.55 & 323.48 & 610.94 \\
\hline & No. 3 Chloroform fraction & $\mathrm{M}_{2} \mathrm{~N}_{3}$ & 2.35 & $Y=-2.30+3.53 X$ & 117.18 & 100.55 & 136.56 \\
\hline & No. 4 Chloroform fraction & $\mathrm{M}_{2} \mathrm{~N}_{4}$ & 1.28 & $Y=-4.88+4.01 X$ & 292.40 & 252.38 & 338.78 \\
\hline & No. 1 Chloroform fraction & $\mathrm{M}_{2} \mathrm{~N}_{1}$ & 3.18 & $Y=-4.37+4.21 X$ & 168.30 & 149.05 & 190.03 \\
\hline \multirow{3}{*}{$\frac{\stackrel{n}{\Xi}}{\stackrel{0}{0}}$} & No. 2 Chloroform fraction & $\mathrm{M}_{2} \mathrm{~N}_{2}$ & 0.68 & $Y=-3.59+3.50 X$ & 283.51 & 240.99 & 333.54 \\
\hline & No. 3 Chloroform fraction & $\mathrm{M}_{2} \mathrm{~N}_{3}$ & 0.411 & $Y=-2.28+3.64 X$ & 100.32 & 84.80 & 118.69 \\
\hline & No. 4 Chloroform fraction & $\mathrm{M}_{2} \mathrm{~N}_{4}$ & 5.07 & $Y=-6.13+4.70 X$ & 233.29 & 179.94 & 302.46 \\
\hline
\end{tabular}


Table VIII. Relative toxicity of different separated bands visualized by UV light and washed with ethyl acetate fraction of Zingiber cassumunar rhizome against Tribolium castaneum adults

\begin{tabular}{|c|c|c|c|c|c|c|c|}
\hline \multirow{2}{*}{$\begin{array}{l}\text { Hours } \\
\text { after } \\
\text { treatment }\end{array}$} & \multirow{2}{*}{$\begin{array}{l}\text { Plant materials } \\
\text { Zingiber cassumunar }\end{array}$} & \multirow[b]{2}{*}{ Fractions } & \multirow{2}{*}{$\begin{array}{l}\chi^{2} \text { values for } \\
\text { heterogeneity }\end{array}$} & \multirow{2}{*}{ Regression equation } & \multirow{2}{*}{$\begin{array}{l}\mathrm{LD}_{50} \\
\left(\mu \mathrm{g} / \mathrm{cm}^{2}\right)\end{array}$} & \multicolumn{2}{|c|}{ Fiducial limits } \\
\hline & & & & & & Lower & Upper \\
\hline \multirow{4}{*}{ 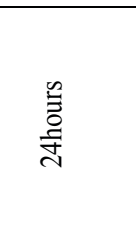 } & No. 1 Ethyl acetate fraction & $\mathrm{M}_{3} \mathrm{~N}_{1}$ & - & - & - & - & - \\
\hline & No. 2 Ethyl acetate fraction & $\mathrm{M}_{3} \mathrm{~N}_{2}$ & - & - & - & - & - \\
\hline & No. 3 Ethyl acetate fraction & $\mathrm{M}_{3} \mathrm{~N}_{3}$ & 2.20 & $Y=-2.27+2.78 X$ & 415.47 & 307.04 & 562.19 \\
\hline & No. 4 Ethyl acetate fraction & $\mathrm{M}_{3} \mathrm{~N}_{4}$ & - & - & - & - & - \\
\hline \multirow{4}{*}{ 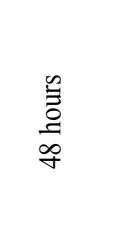 } & No. 1 Ethyl acetate fraction & $\mathrm{M}_{3} \mathrm{~N}_{1}$ & 2.23 & $\mathrm{Y}=-2.79+3.19 \mathrm{X}$ & 277.51 & 233.19 & 330.25 \\
\hline & No. 2 Ethyl acetate fraction & $\mathrm{M}_{3} \mathrm{~N}_{2}$ & 0.007 & $Y=2.81+0.596 X$ & 4563.90 & 38.99 & 534116.30 \\
\hline & No. 3 Ethyl acetate fraction & $\mathrm{M}_{3} \mathrm{~N}_{3}$ & 11.79 & $Y=-2.91+3.49 X$ & 185.22 & 115.35 & 297.41 \\
\hline & No. 4 Ethyl acetate fraction & $\mathrm{M}_{3} \mathrm{~N}_{4}$ & - & - & - & - & - \\
\hline \multirow{4}{*}{$\begin{array}{l}\stackrel{2}{\Xi} \\
\stackrel{\Xi}{\pi}\end{array}$} & No. 1 Ethyl acetate fraction & $\mathrm{M}_{3} \mathrm{~N}_{1}$ & 3.18 & $\mathrm{Y}=-4.37+4.21 \mathrm{X}$ & 168.30 & 149.05 & 190.03 \\
\hline & No. 2 Ethyl acetate fraction & $\mathrm{M}_{3} \mathrm{~N}_{2}$ & 3.96 & $\mathrm{Y}=1.61+1.33 \mathrm{X}$ & 357.08 & 127.71 & 998.38 \\
\hline & No. 3 Ethyl acetate fraction & $\mathrm{M}_{3} \mathrm{~N}_{3}$ & 6.12 & $Y=-2.54+3.44 X$ & 154.54 & 108.93 & 219.25 \\
\hline & No. 4 Ethyl acetate fraction & $\mathrm{M}_{3} \mathrm{~N}_{4}$ & - & - & - & - & - \\
\hline
\end{tabular}

Table IX. Relative toxicity of different separated bands visualized by UV light and washed with methanol fraction of Zingiber cassumunar rhizome against Tribolium castaneum adults

\begin{tabular}{|c|c|c|c|c|c|c|c|}
\hline \multirow{2}{*}{$\begin{array}{l}\text { Hours } \\
\text { after } \\
\text { treatment }\end{array}$} & \multirow{2}{*}{$\begin{array}{l}\text { Plant materials } \\
\text { Zingiber cassumunar }\end{array}$} & \multirow[b]{2}{*}{ Fractions } & \multirow{2}{*}{$\begin{array}{l}\chi^{2} \text { values for } \\
\text { heterogeneit } \\
\text { y }\end{array}$} & \multirow[b]{2}{*}{ Regression equation } & \multirow{2}{*}{$\begin{array}{l}\mathrm{LD}_{50} \\
\left(\mu \mathrm{g} / \mathrm{cm}^{2}\right)\end{array}$} & \multicolumn{2}{|c|}{ Fiducial limits } \\
\hline & & & & & & Lower & Upper \\
\hline \multirow{4}{*}{$\begin{array}{l}\stackrel{\infty}{\leftrightarrows} \\
\stackrel{\Xi}{J} \\
\text { ন }\end{array}$} & No. 1 Methanol fraction & $\mathrm{M}_{4} \mathrm{~N}_{1}$ & - & - & - & - & - \\
\hline & No. 2 Methanol fraction & $\mathrm{M}_{4} \mathrm{~N}_{2}$ & - & - & - & - & - \\
\hline & No. 3 Methanol fraction & $\mathrm{M}_{4} \mathrm{~N}_{3}$ & 0.13 & $Y=-2.51+2.99 X$ & 326.13 & 263.08 & 404.28 \\
\hline & No. 4 Methanol fraction & $\mathrm{M}_{4} \mathrm{~N}_{4}$ & - & - & - & - & - \\
\hline \multirow{4}{*}{$\begin{array}{l}\stackrel{\infty}{\Xi} \\
\stackrel{\Xi}{\Xi} \\
\stackrel{\infty}{+}\end{array}$} & No. 1 Methanol fraction & $\mathrm{M}_{4} \mathrm{~N}_{1}$ & - & - & - & - & - \\
\hline & No. 2 Methanol fraction & $\mathrm{M}_{4} \mathrm{~N}_{2}$ & - & - & - & - & - \\
\hline & No. 3 Methanol fraction & $\mathrm{M}_{4} \mathrm{~N}_{3}$ & 1.18 & $Y=-4.02+3.84 X$ & 223.25 & 195.82 & 254.51 \\
\hline & No. 4 Methanol fraction & $\mathrm{M}_{4} \mathrm{~N}_{4}$ & - & - & - & - & - \\
\hline \multirow{4}{*}{$\begin{array}{l}\frac{n}{\Xi} \\
\frac{\Xi}{\pi} \\
\text { त }\end{array}$} & No. 1 Methanol fraction & $\mathrm{M}_{4} \mathrm{~N}_{1}$ & - & - & - & - & - \\
\hline & No. 2 Methanol fraction & $\mathrm{M}_{4} \mathrm{~N}_{2}$ & - & - & - & - & - \\
\hline & No. 3 Methanol fraction & $\mathrm{M}_{4} \mathrm{~N}_{3}$ & 0.01 & $Y=-4.63+4.42 X$ & 151.21 & 134.39 & 170.14 \\
\hline & No. 4 Methanol fraction & $\mathrm{M}_{4} \mathrm{~N}_{4}$ & - & - & - & - & - \\
\hline
\end{tabular}


(2008) also reported that petroleum ether extracts of $Z$. cassumunar leaf and rhizome showed highest repellency to T. castaneum than those of other extracts (Methanol and Acetone) tested. Result of the present study is in agreement with the results of Somboom and Co-workers (Somboom and Pimsamarn 2011), who reported that volatile oils from plants of zingiberaceae family, obtained by hydro-distillation method caused tremendous toxicity to rice weevil and flour weevil. The $\mathrm{LC}_{50}$ values at 48 hours were 10543 and 13693ppm respectively. Suthisut and Co-workers (Suthisut et al., 2011) got effective fumigant toxicity of essential oils from rhizomes of zingiberaceae (Alpinia conchigera, Zingiber aerumbet Curcuma zedoaria and their major compounds) against Sitophilus zeamais, Tribolium castaneum, Anisopteromalus calandrae and Trichogramma deion larvae. They also reported that $A$. conchigera oils were toxic to $S$. zeamis, $T$. castaneum and $T$. deion. The $\mathrm{LD}_{50}$ values of $A$. conchigera oils $85 \mu \mathrm{l} / \mathrm{L}$ and $73 \mu \mathrm{l} / \mathrm{L}$ after 48 hours exposure time against $S$. zeamais and $T$. castaneum adults respectively. T. castaneum was more susceptible than $S$. zeamais to the eight pure compounds.

The present results supported the finding of Nugroho and Co-workers (Nugroho et al., 1996) who reported that extracts from rhizomes of Kaempferia rotunda and Zingiber cassumunar displayed significant insecticidal activity in chronic feeding bioassays against neonate larvae of Spodoptera littoralis. They also reported the presence of two phenylbutanoids compounds in $Z$. cassumunar rhizome, which had $L_{50}$ values 121 and $127 \mathrm{ppm}$ respectively against neonate larvae of $S$. littoralis. Talukder and Co-workers (Talukder et al., 2009) reported that the emulsifed products of petroleum ether extract of Acorous calamus combined with Zingiber cassumunar exhibited moderate effect against Callosobruchus chinensis, Sitophilus oryzae and Tibolium castaneum adults. They also reported that the $\mathrm{LD}_{50}$ values of emulsified products were 547.08 and $452.51 \mu \mathrm{g} / \mathrm{cm}^{2}$ after 24 and 48 hours exposure time respectively.

\section{Conclusion}

The phytochemical study of the plant extracts reveal that there used in successful control of noxious insects. It may be used as alternative to synthetic insecticides. Petroleum ether extract of $Z$. cassumunar rhizome were most effective against $T$. castaneum adult than acetone and methanol extract. The result also revealed that among the fractions of petroleum ether extract of $Z$. cassumunar obtained from colum chromatography $\left(\mathrm{A}_{1}\right)(\mathrm{MN}-1, \mathrm{MN}-2, \mathrm{MN}-3, \mathrm{MN}-4$, $\mathrm{MN}-5, \mathrm{MN}-6, \mathrm{MN}-7$ and $\mathrm{MN}-8) \mathrm{MN}-1$ fraction again was subjected to Preparative Thin Layer Chromatography (PTLC). Four fractions were found (M-1, M-2, M-3, M-4) by the use of UV-light $(350 \mathrm{~nm})$. These fractions washed with chloroform and ethyl acetate showed the most effective result than other solvent against $T$. castaneum adults. The insecticidal property of the Z. cassumunar extract may be due to the presence of Phenolic compounds. There is a need to conduct farther study on above mentioned fraction against other stored product pests to establish its efficacy as plant based insecticide.

\section{Acknowledgement}

The authors express their thanks to the concerned authority and Director, BCSIR, Laboratories, Rajshahi for providing research facilities. The authors are also grateful to the Chairman, Department of Chemistry, University of Rajshahi, for providing necessary laboratory facilities.

\section{References}

Abbott, WS (1925), A method of computing the effectiveness of an insecticide. J. econ. Ent. 18: 265-267.

Bandra, KAMP, Kumar V, Saxena RC and Ramdas, PK (2005), Bruchid (Coleoptera: Bruchidae) Ovicidal Phenylbutanoid from Zingiber purpureum. J. econ. Ent. 98(4): 1163-1169.

Bhuiyan, M N I, Chowdhury J U And Begum J (2008), Volatile constituents of essential oils isolated from leaf and rhizome of Zingiber cassumunar Roxb. Bangladesh J. Pharmacol. 3: 69-73

Busvine, JR (1971) A Critical Review of the Techniques for Testing Insecticides. Commonwealth Agricultural Bureau, London. (1971) pp. 345

Chairul, Praptiwi and Chairul, Sofnie Marusin (2009), Phagocytosis Effectivity Test of Phenylbutenoid Compounds Isolated from Bangle (Zingiber cassumunar Roxb.) Rhizome. Biodiversitas Vol. 10 (1): $40-43$

Giwanon R, Thubthimthed S, Rerkam U and Sunthorntanasart, T. (2000), Antimicrobial activity of terpinen-4-ol and sabinene. Thai J Pharm Sci. 24 (Suppl): 27.

Iswantini D, Silitonga, R F, Martatilofa E and Darusman L K (2011), Zingiber cassumunar, Guazuma ulmifolia, and Murraya paniculata Extracts as Antiobesity: In Vitro Inhibitory Effect on Pancreatic Lipase Activity. HAYATI J Biosci. Vol. 18(1): p. 6-10 
Kamazeri TSAT, Samah OA, Taher M, Susanti D, Qaralleh H. (2012), Antimicrobial activity and essential oils of Curcuma aeruginosa, Curcuma mangga, and Zingiber cassumunar from Malaysia. Asian Pac J Trop Med 5(3): 202-209.

Khanam, L A M, Ranman M S and Mahfuz I (2008), Repellency of Tribolium castaneum Herbst and Tribolium confusum Duval (Coleoptera : Tenebrionidae) to the Rhizome and leaf extracts Zingiber cassumunar Roxb. Bangladesh j. Sci. Ind. Res. 43(2): 251-258.

Khanam, L A Talukder D and Ahmed K N (2005), Pesticidal action of some plant materials against Sitophilus oryzae (L.) Bangladesh J. Sci. Ind. Res. 40 (3-4): 203-210.

Khanam L A M Talukder D and Dey K C (2006), Bioactivity of some plant extracts against the rice weevil, Sitophilus oryzae. J. Asiat. Soc, Bangladesh Sci. 32(2): 219-226.

Nugroho BW, Schwarz B, Wray V and Proksch P (1996), Insectididal constituents from rhizomes of Zingiber cassumunar and Kaempferia rotunda. Phytochemistry 41(1): 129-132.

Park T, Mertz, DB and Petrusewich, M (1961), Genetic strains of Tribolium: their primary characteristics. Physiol. Zool., 34: 62-80.

Pithayanukul, $\mathrm{P}$, Tubprasert $\mathrm{J}$ and WuthiUdomlert $\mathrm{M}$ (2008), In Vitro Antimicrobial Activity of Zingibercassumunar (Plai) Oil and a 5\% Plai Oil Gel. Phytother. Res. 21: 164-169.

Somboom S and Pimsamarn S (2011), Potential of using zingiberous plant volatile oils for flour weevil ( Tribolium castaneum Herbst) and rice weevil (Sitophilus oryzae L.) control. Agricultural Science Journal. 34(4-6(Suppl.)): 183-186.
Sukatta U, Rugthaworn P, Punjee P, Chidchenchey S and Keeratinijakal V (2009), Chemical Composition and Physical Properties of Oil from Plai (Zingiber cassumunar Roxb.) Obtained by Hydro Distillation and Hexane Extraction. Kasetsart J. (Nat. Sci.) 43: $212-217$.

Suthisut D, Fields PG and Chandrapatya A (2011), Fumigant toxicity of essential oils from three Thai plants (Zingiberaceae) and their major compounds against Sitophilus zeamais, Tribolium castaneum and two parasitoids. J. Stored Prod. Res., 47: 222-230.

Talukder D and Khanam L A M (2011), The fumigant toxicity of four plant based products against three stored product pests. Int. J. Sustain. Crop Prod. 6(1): 6-9.

Talukder D and Khanam L A M (2009), Toxicity of four plant based products against three stored product pests J. bio-sci. 17: 149-153.

Tripathi P, Dubey NK and Shukla AK (2008), Use of some essential oils as post-harvest botanical fungicides in the management of grey mould of grapes caused by Botrytis cinerea. World J. Microbiol. Biotechnol. 24: 39-46.

Wanauppathamkul S (2003), Plaitanoids.1 ed. The Innovation Development Fund, National Science and Technology Development Agency, Ministry of Science and Technology, Bangkok. pp. 40.

Yanbin Lu, Cuirong Sun, Yu Wang, Yuanjiang Pan (2005), Preparative isolation and purification of two phenylbutenoids from the rhizomes of Zingiber Cassumunar by upright counter-current chromatography. J. Chromatogr. A Vol. 1089(1-2): 258-262.

Received: 11 May 2014; Revised: 25 January 2015; Accepted: 24 March 2015. 